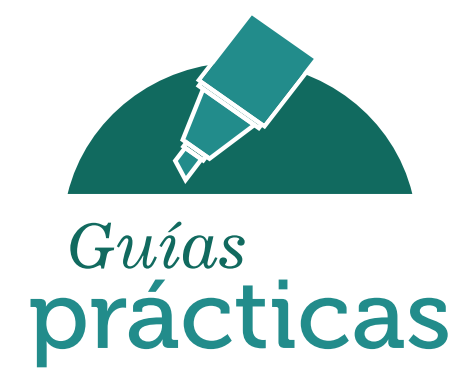

\title{
EXPLORACIÓN DE LOS SIGNOS VITALES
}

Alberto Mendivil de la Ossa

Lina María Gómez Duque

Universidad Cooperativa de Colombia Sede Medellín 


\section{ACERCA DE LOS AUTORES}

José Alberto Mendivil de la Ossa, médico, magíster en Epidemiología. Profesor de tiempo completo, Facultad de Medicina, Universidad Cooperativa de Colombia, Sede Medellín. Correo electrónico:

jose.mendivil@campusucc.edu.co

CVLAC:

https://scienti.minciencias.gov.co/cvlac/ visualizador/generarCurriculoCv.do?cod_ $r h=0001426532$.

ORCID: https://orcid.org/0000-0001-9006-8866

Google Scholar:

https://scholar.google.com/citations?user $=$ V-b414sAAAAJ\&hl=es

Lina María Gómez Duque, médica y cirujana, magíster en Educación. Profesora de tiempo completo, Facultad de Medicina, Universidad Cooperativa de Colombia, Sede Medellín.

Correo electrónico:

lina.gomezdu@campusucc.edu.co

\section{CÓMO CITAR ESTE DOCUMENTO}

Mendivil de la Ossa JA, Gómez Duque LM. Exploración de los signos vitales (Generación de contenidos impresos N. $\left.{ }^{\circ} 25\right)$. Ediciones Universidad Cooperativa de Colombia, 2021. https://doi.org/10.16925/gcgp.41

\section{NOTA LEGAL}

El presente documento de trabajo ha sido incluido dentro de nuestro repositorio institucional como Apropiación social de conocimiento por solicitud del autor, con fines informativos, educativos o académicos. Asimismo, los argumentos, datos y análisis incluidos en el texto son responsabilidad absoluta del autor y no representan la opinión del Fondo Editorial o de la Universidad.

\section{DISCLAIMER}

This coursework paper has been uploaded to our institutional repository as Social Appropriation of Knowledge due to the request of the author. This document should be used for informational, educational or academic purposes only. Arguments, data and analysis included in this document represent authors' opinion not the Press or the University.

(c) (1) () Este documento puede ser consultado, descargado o reproducido desde nuestro repositorio institucional (http://repository. ucc.edu.co/handle/20.500.12494/7369) para uso de sus contenidos, bajo la licencia de Creative Commons Reconocimiento-NoComercial-SinObraDerivada 4.0 Internacional. http://creativecommons.org/licenses/by-nc-nd/4.0/ 


\section{TABLA DE CONTENIDO}

Introducción 5

Delimitación de la guía de práctica 5

$\begin{array}{ll}\text { Recomendaciones de uso de esta guía } & 6\end{array}$

Marco teórico 6

Medición de la temperatura $\quad 6$

$\begin{array}{ll}\text { Medición de la frecuencia cardiaca } & 7\end{array}$

Medición del pulso $\quad 7$

$\begin{array}{ll}\text { Medición de la frecuencia respiratoria } & 7\end{array}$

Medición de la presión arterial $\quad 8$

Medición de la oximetría $\quad 9$

Objetivos, materiales y reglamento 10

$\begin{array}{ll}\text { Objetivos } & 10\end{array}$

Materiales 10

$\begin{array}{ll}\text { Reglamento } & 10\end{array}$

Descripción de las actividades y procedimientos 11

$\begin{array}{ll}\text { Referencias } & 12\end{array}$ 


\title{
25 EITALLRA
}

\author{
José Alberto Mendivil de la Ossa
}

Lina María Gómez Duque

\section{Resumen}

Este documento parte de los cursos Semiología del Paciente Sano y Semiología Clínica y se desarrolló con base en los textos guía de sus correspondientes syllabus: Manual Seidel de exploración física y Semiología médica y técnica exploratoria. Comprende los aspectos básicos de la semiotecnia y la interpretación de los hallazgos semiológicos del examen de los signos vitales, siguiendo este orden: 1) evaluación de la temperatura corporal, utilizando distintos tipos de termómetros, teniendo como referencia distintas zonas del cuerpo donde pueda ser medida la temperatura; 2) evaluación de la frecuencia respiratoria, a través de la observación o palpación, de acuerdo con el estado del paciente; 3) evaluación de la frecuencia cardiaca y el pulso, utilizando la palpación para el pulso en arterias de múltiples regiones del cuerpo, y acompañarla de la descripción de las características del pulso, así como la auscultación de la frecuencia cardiaca y correlación de la asociación entre frecuencia cardiaca y pulso si está presente; 4) evaluación de la presión arterial, revisando primeramente la condición del paciente para esta medición y luego aplicando correctamente el uso del tensiómetro y estetoscopio. Se inicia con la técnica palpatoria para completar la medición con la técnica auscultatoria; esto con el paciente en varias posiciones e igualmente en ambas extremidades superiores a nivel de antebrazo y muñeca; por último, 5) evaluación de la oximetría de pulso a través de un pulsioxímetro, cuantificando los valores e interpretando sus resultados, además de información adicional de relevancia como la onda de pulso y la temperatura o perfusión del sitio en el que se toma la medición.

Palabras clave: evaluación, frecuencia cardiaca, frecuencia respiratoria, oximetría de pulso, presión arterial, temperatura corporal. 


\section{INTRODUCCIÓN}

La evaluación de los signos vitales constituye el primer aspecto del examen físico del médico; comprende la medición de la temperatura corporal, de la frecuencia cardiaca y pulso, de la frecuencia respiratoria, de la presión arterial sistémica y, por último, de la oximetría de pulso. Todas estas mediciones brindan una visión panorámica de la homeostasis del paciente y permiten definir algunos desequilibrios que clasificarán el estado del paciente, por ejemplo, como crítico.

La evaluación de la temperatura es importante al ser un signo de inflamación presente en muchas condiciones anómalas; se ejecutará en varias regiones del cuerpo y se abordará el factor de corrección para tener un valor más aproximado a la temperatura central. Por su parte, en la evaluación de la frecuencia cardiaca y del pulso se abordará la correcta medición de estos y la relación que debe haber entre el uno y el otro, como ejemplo del buen gasto cardiaco y la poscarga. En esta práctica se hará uso de múltiples arterias del cuerpo en las que pueden ser evaluados el pulso y sus características.

La valoración de la frecuencia respiratoria nos permite conocer el número de respiraciones por minuto y también el patrón respiratorio del paciente, por lo que debe ser precisamente relacionada con el aprendizaje de los aspectos normales del ciclo respiratorio y de los hallazgos anómalos en él que permiten clasificarlo de distinta manera, por ejemplo, respiración de Biot, Cheyne-Stokes, Kussmaul, entre otros.

La oximetría será tomada como un signo auxiliar para conocer el estado del intercambio gaseoso a nivel respiratorio, respetando siempre los límites de la prueba, pues, dado que hay condiciones extrapulmonares que pueden alterarla, el estudiante de medicina debe ser capaz de identificar qué condiciones alterarán esta medición, para así dar una correcta interpretación.

Para la ejecución de esta práctica, es imperativo que el estudiante conozca los rangos de normalidad de los signos vitales en distintos grupos etarios, además de la clasificación de estos según los valores obtenidos, de tal manera que sea capaz de clasificar al paciente como "sano" o su estado clínico en cualquier otro caso de anormalidad.

\section{Delimitación de la guía de práctica}

Esta guía pretende favorecer el aprendizaje adecuado de la medición de signos vitales, a partir del cual el estudiante pueda ser capaz de:

1. Evaluar la temperatura corporal con el uso de diferentes tipos de termómetros y en diferentes regiones del cuerpo, teniendo en cuenta la corrección de esta cuando sea necesario.

2. Cuantificar la frecuencia respiratoria adecuadamente a través de la observación del patrón respiratorio del paciente.

3. Cuantificar la frecuencia cardiaca y el pulso a través de la auscultación y palpación, utilizando diferentes arterias en la anatomía y discriminando las características del ciclo cardiaco y del pulso.

4. Medir la presión arterial sistémica a través de la combinación de la técnica palpatoria y auscultatoria, haciendo uso de las condiciones adecuadas para esta medición y con la correcta interpretación de esta, considerando la corrección de los valores cuando sea pertinente.

5. Evaluar e interpretar los parámetros obtenidos a través de la oximetría de pulso con respecto a la condición actual del paciente. 


\section{Recomendaciones de uso de esta guía}

Para obtener el máximo provecho de esta guía, es esencial que tenga en cuenta las siguientes recomendaciones en su orden:

1. Siga la descripción de procedimientos al pie de la letra, evitando saltar pasos aun cuando considere que alguna de las indicaciones ya es de su dominio. Recuerde que esta guía le explica cómo es el proceso completo para el examen físico de un órgano o región bajo una técnica adecuada, buscando sacar el mayor provecho del examen físico del paciente.

2. Conserve las indicaciones con respecto a su ubicación con relación al paciente, distancias, uso de los instrumentos, técnica por realizar, entre otras, pues de esta manera se asegura que el procedimiento sea seguro en el marco de la bioseguridad, la responsabilidad del acto médico y, además, que este sea correcto para obtener los hallazgos más fidedignos posibles.

3. Respete la indicación de la guía sobre la práctica en humanos o en dispositivos de simulación; en algunos puntos la guía será explicita sobre su aplicación en otras personas; de lo contrario, utilice el material de simulación; algunos procedimientos pueden ser peligrosos o, incluso, pueden generar la percepción de vulneración en la persona en la que se ejecuta la técnica. En todo caso, recuerde pedir permiso antes de hacer cualquier maniobra o técnica semiológica sobre otra persona.

4. Para comprender adecuadamente la guía, el estudiante debe tener conocimientos previos de la semiología médica, por lo que se debe complementar con una lectura previa o de una clase magistral sobre el tema. Igualmente, luego de cada actividad práctica, se recomienda a los estudiantes el repaso de la interpretación de los posibles hallazgos derivados de las técnicas descritas en la guía.

\section{MARCO TEÓRICO}

La evaluación de los signos vitales comprende la valoración de la temperatura, el pulso, la frecuencia cardiaca, la frecuencia respiratoria y la presión arterial, como se explica a continuación.

\section{Medición de la temperatura}

Para la toma de la temperatura, se utilizarán termómetros de mercurio y termómetros electrónicos o digitales.

- Sitio de la toma: si está utilizando un termómetro digital, se puede colocar en la axila, el oído o la zona sugerida por el fabricante. Para el termómetro de mercurio se pueden utilizar varios sitios de medición (rectal, oral, axilar) [1]. Para esta práctica se usará la región axilar.

- Técnica para la medición: para tomar la temperatura con el termómetro digital, se procede a encender el termómetro, se espera a que se ponga en cero. El estudiante procederá a colocar el termómetro en la región indicada, ya sea en la axila o el oído. Se espera hasta que el termómetro produzca un sonido que indicará que ya se realizó la medición, se mira en la pantalla la temperatura del paciente. Para tomar la temperatura con el termómetro de mercurio, el estudiante lo sujetará por el extremo contrario a donde está el bulbo de mercurio; verificará que la columna de mercurio esté por debajo de los $35^{\circ} \mathrm{C}$; de lo contrario, dará unas sacudidas al termómetro hasta que baje; colocará el termómetro en la axila del paciente y esperará de 3 a 5 minutos, después de los cuales procederá a 
leer la temperatura del paciente; si la temperatura se ha tomado en la región axilar, al resultado se le deben sumar $0,5^{\circ} \mathrm{C}$ [2].

- Interpretaciones: la temperatura normal es de $36,5^{\circ} \mathrm{C}$ a $37,5^{\circ} \mathrm{C}$. Si la temperatura está entre $37,5^{\circ} \mathrm{C}$ y $38^{\circ} \mathrm{C}$, se considera una febrícula. Si la temperatura se encuentra entre $38^{\circ} \mathrm{C}$ y $39^{\circ} \mathrm{C}$, se considera fiebre moderada. Finalmente, si la temperatura se encuentra por encima de $39^{\circ} \mathrm{C}$, fiebre alta [3].

\section{Medición de la frecuencia cardiaca}

- Sitio de la toma: para la evaluación de la frecuencia cardiaca, esta solo puede ser auscultada en el precordio; sin embargo, se debe contrastar siempre con el pulso para observar su ritmo y asociación [3].

- Técnica para la medición: con la membrana o campana del estetoscopio, se aplica esta sobre cualquier foco de auscultación cardiaca, a la vez que se toma el pulso en alguna arteria, preferiblemente una de las arterias radiales. Se mide el número de latidos por 60 segundos y se contrasta que estos sean concordantes con las ondas de pulso [1].

- Interpretaciones: para la interpretación del rango de valor de frecuencia cardiaca, el estudiante debe remitirse a las tablas con los valores de referencia para cada grupo etario.

\section{Medición del pulso}

- Sitio de la toma: la medición del pulso arterial se puede realizar en cualquiera de los sitios conocidos para esta, generalmente donde una arteria se pueda comprimir sobre una superficie ósea (temporal, carotídeo, braquial, radial, cubital, femoral, poplíteo, tibial posterior, pedio) [1]; sin embargo, el sitio más común y el cual se usará será el pulso radial. Si hay contraindicación o limitaciones para este, se tomará en otro de los lugares anotados.

- Técnica para la medición: el estudiante tomará la muñeca del paciente con su mano, colocando el dedo pulgar en el dorso de la muñeca y el pulpejo de los dedos (segundo, tercero y cuarto) sobre la arteria radial y contará las pulsaciones por el lapso de un minuto completo, después de lo cual describirá las características del pulso (frecuencia, ritmo, amplitud, igualdad y simetría); para esto deberá comparar el pulso en ambas muñecas. Esta técnica aplica también para la toma de pulsos en otras localizaciones.

- Interpretaciones: si las pulsaciones están por debajo del rango de normalidad, se hablará de bradisfigmia (bradicardia) si concuerda con la frecuencia cardiaca; y si están por encima del valor de normalidad se tendrá una taquisfigmia (taquicardia), en caso de que concuerde con la frecuencia cardiaca. Es importante aclarar que el término estricto sería bradisfigmia o taquisfigmia, pero en la práctica, por facilidad, se habla de bradicardia o taquicardia, teniendo en cuenta que pulso y frecuencia cardiaca concuerden; en caso contrario habrá un cuadro de disociación entre la frecuencia cardiaca y pulso. Siempre se deben conocer los valores de normalidad según la edad del paciente [3].

\section{Medición de la frecuencia respiratoria}

La medición de la frecuencia respiratoria puede llegar a ser bastante sencilla en condiciones de normalidad; sin embargo, cuando hay una respiración anómala, contar la frecuencia de ciclos por minuto puede llegar a ser bastante difícil y requerir mucha concentración del evaluador en la referencia que se usa para medirla: 
- Sitio de la toma: la frecuencia respiratoria debe ser evaluada directamente en el tórax, siendo más confiable así que teniendo como referencia cualquier otra fuente.

- Técnica para la medición: se debe evitar a toda costa que el paciente sepa que se le está midiendo la frecuencia respiratoria, pues es probable que intente modificarla, sea para disminuirla o aumentarla; por ello, evitaremos tocar al paciente en el tórax o auscultarlo de ser posible [3]. Una buena forma de distraer el paciente es hacer la técnica para medir el pulso en una muñeca, para que así crea que estamos evaluando esto.

La primera forma para contar los ciclos respiratorios es observar el movimiento del tórax del paciente, lo que nos permite evaluar el número de inhalaciones y exhalaciones en 60 segundos; esto es a veces muy difícil, dado que el paciente puede tener una respiración bastante superficial o poco notable, por ejemplo, por el uso de ropa ancha. Esta es la mejor técnica en un paciente con un patrón de respiración que no esté aparentemente alterado [3].

Una segunda forma consiste en apoyar las manos sobre el tórax anterior o posterior y contar el número de ciclos; evidentemente esto puede hacer que el paciente sea consciente de que estamos evaluando su respiración; por ello, esta técnica es más adecuada en casos en los que el paciente tiene un patrón respiratorio alterado, pues es prácticamente independiente a su voluntad [3].

Una última forma es sencillamente auscultar los ruidos respiratorios y hacer el conteo correspondiente en un minuto. Funciona mejor en los mismos casos que en la técnica anterior.

- Interpretaciones: el estudiante debe remitirse a las tablas de frecuencia respira- toria según edades para poder identificar los rangos de normalidad según este criterio. Sin embargo, siempre se podrá llegar a la misma clasificación: si los ciclos en un adulto están por debajo del rango de normalidad, se hablará de bradipnea, y si están por encima del valor de normalidad, se tendrá una taquipnea. Los términos hipopnea e hiperpnea se refieren, respectivamente, a la reducción o aumento de flujo de aire en la inspiración, situación que podemos evaluar en la profundidad de los movimientos respiratorios, pero no en la frecuencia respiratoria. Por tanto, ambas parejas de términos deben ser bien distinguidas a la hora de describir los hallazgos [1].

\section{Medición de la presión arterial}

- Sitio de la toma: la presión arterial se mide generalmente en los brazos, con el paciente sentado.

- Técnica para la medición: con el brazo descubierto, se debe colocar el manguito del tensiómetro alrededor de este, 2 o 3 $\mathrm{cm}$ por encima del pliegue del codo, verificando que no quede muy suelto ni muy apretado, pues esto puede alterar las cifras de la presión arterial. Se debe realizar de manera palpatoria y auscultatoria. Con el método palpatorio solo se podrá medir la presión sistólica de la siguiente manera: con el manguito colocado en el brazo, se procede a buscar el pulso radial; cuando se encuentra el pulso y con el miembro superior elevado hasta la altura del corazón, se precederá a insuflar, hasta que note que desaparece el pulso; luego de esto se insuflará 20 mmHg más, después de lo cual se empieza a desinflar de a 1 o $2 \mathrm{~mm}$, con el fin de poder percibir exactamente el momento en el que el pulso aparece de nuevo; en este punto se define que se encuentra la presión sistólica [1]. 
Luego de esto, se desinfla totalmente el manguito y se espera un momento. Se procede entonces a realizar la técnica auscultatoria, en la cual se coloca el fonendoscopio en el pliegue del codo; sobre el trayecto de la arteria humeral, se insufla hasta 20 mmHg más de lo que le dio la presión sistólica palpatoria, momento en que se empieza a desinflar entre 1 a $3 \mathrm{mmHg}$. Cuando se empiece a escuchar un sonido, esa será la presión sistólica. Se continuará desinflando entre 1 y $3 \mathrm{mmHg}$ hasta que desaparezcan los sonidos; en este momento se puede definir la presión diastólica. En algunos pacientes se puede continuar escuchando sonidos hasta casi llegar a 0; en estos casos hay un momento en que los ruidos sufren un cambio en la intensidad, ahí se tomará como la presión diastólica [1].

- Interpretaciones: para realizar la interpretación con el resultado de las cifras de presión arterial, el estudiante debe remitirse a las cifras de clasificación de estadios de la presión arterial vigentes en el momento. Generalmente se podrá definir que el paciente está hipotenso si está por debajo de los valores promedio según los criterios de clasificación por edad, sexo, antecedente de hipertensión u otros. Se clasificará con hipertensión alta o hipertensión también según los criterios utilizados y la clasificación para ese momento [2].

\section{Medición de la oximetría}

La oximetría nos permite conocer un valor aproximado de la saturación de oxígeno que lleva la hemoglobina del paciente; por ello, es importante antes entender que una buena saturación de oxígeno no significa que la circulación de este gas en el cuerpo sea normal, dado que dependerá de su unión y separación de la hemoglobina, por lo que la cantidad de esta y su curva de disociación modificarán de gran manera el comportamiento de este gas para el intercambio en los tejidos [4].
Además de estos, otros factores que afectan la medición de la saturación de oxígeno son la temperatura corporal, pues la vasoconstricción generada por el frío disminuye la medición, así como el uso de esmaltes en las uñas, entre otros [1].

- Sitio de la toma: se puede utilizar cualquier dedo de la mano; preferiblemente, por la forma del oxímetro, usar el más largo. En niños se pueden usar oxímetros especiales que envuelven una parte del pie.

\section{- Técnica para la medición:}

- Calentar la extremidad a usar antes, envolviéndola en una manta, por ejemplo.

- Si los dedos tienen esmalte, retirar este con un solvente adecuado.

- Se inserta el dedo en el oxímetro y se enciende después de insertado el dedo, esto facilita que capte más rápido la onda de pulso.

- Observar el resultado.

- Interpretaciones: la saturación de oxígeno puede variar con muchas condiciones, pero en un paciente sano de cualquier edad, usualmente un valor normal estará por encima de 90\%; sin embargo, valores menores son tolerables en personas con enfermedad pulmonar que requieren más del control del $\mathrm{CO}_{2}$ que del oxígeno para sostener su respiración, o valores más altos que pueden incluso ser indeseables en caso de neonatos prematuros con riesgo de retinopatía de la prematuridad.

Además de lo anterior, se debe tener en cuenta la información adicional que brinda el oxímetro, como son la onda y el pulso, con los que valoraremos si la medición es buena, pues una onda pobre con un pulso que no concuerda con el paciente puede hacernos sospechar de una medición errada. 


\section{OBJETIVOS, MATERIALES Y REGLAMENTO}

\section{Objetivos}

1. Describir los prerrequisitos y la técnica adecuada para la medición de la temperatura corporal, frecuencia cardiaca, pulso, presión arterial y oximetría de pulso.

2. Ejecutar correctamente la técnica para medición de la temperatura corporal, frecuencia cardiaca, pulso, presión arterial y oximetría de pulso.

3. Analizar las condiciones clínicas del paciente que pueden modificar medición de la temperatura corporal, frecuencia cardiaca, pulso, presión arterial y oximetría de pulso.

4. Interpretar correctamente, y con relación a la condición clínica del paciente, las mediciones de la temperatura corporal, frecuencia cardiaca, pulso, presión arterial y oximetría de pulso.

\section{Materiales}

1. Bata blanca

2. Uniforme

3. Estetoscopio

4. Tensiómetro (fijo o portátil)

5. Termómetros (de mercurio, digital, de infrarrojos)

6. Oxímetro de pulso

7. Algodón

8. Alcohol antiséptico

\section{Reglamento}

1. Los estudiantes deben presentarse máximo 10 minutos antes al ambiente práctico de aprendizaje (APA) asignado para la actividad, de tal forma que se reciban las instrucciones de ingreso para esta.

2. La hora de ingreso a la actividad es la programada por el docente, quien se reservará admitir el ingreso a la actividad en caso de que esta ya haya iniciado.

3. Los estudiantes se comprometen a realizar las actividades prácticas bajo el reglamento de uso del APA requerido.

4. No deben consumirse alimentos durante la actividad.

5. El permiso para grabación de material audiovisual durante la actividad será considerado por el docente; los estudiantes no deberán generar registros fotográficos, de audio, video o transmisiones a través de internet o telefonía sin dicho permiso.

6. Los estudiantes deben portar la bata blanca durante toda la actividad, salvo que, por algún requerimiento de esta, el docente indique que pueden retirársela.

7. Para los procedimientos que se requieran en la actividad práctica, los estudiantes podrán hacer uso de sus propios instrumentos, como son termómetro, estetoscopio, tensiómetro, equipo de órganos, entre otros.

8. La manipulación de los simuladores y la ejecución de procedimientos descritos en esta práctica se llevarán a cabo con la instrucción y vigilancia del docente. No estará permitido, durante la actividad, que los estudiantes realicen otros ejercicios distintos a los descritos o manipular el material de simulación de forma independiente. 
9. En caso de ser requerido, los estudiantes harán uso del equipo de bioseguridad solicitado, sean guantes, mascarilla facial, monogafas u otros.

10. Una vez terminada la actividad, los estudiantes devolverán en su totalidad el material de simulación y tanto el APA como las estaciones de simulación deberán ser entregadas en el estado en que se recibieron y en orden.

\section{DESCRIPCIÓN DE LAS ACTIVIDADES Y PROCEDIMIENTOS}

Para la ejecución de la actividad, los estudiantes deben organizarse primero en parejas; en caso de ser un grupo impar, el número máximo de estudiantes será de tres. Cada grupo debe tener disponible al menos un elemento de los requeridos en materiales y métodos para poder ejecutar adecuadamente la actividad práctica:

1. Evaluación de la temperatura corporal:

a. Medición de la temperatura corporal a nivel axilar. b. Medición de la temperatura corporal a nivel frontal.

2. Evaluación de la frecuencia respiratoria:

a. Conteo de la frecuencia respiratoria.

b. Valoración del patrón respiratorio.

3. Evaluación de la frecuencia cardiaca y el pulso:

a. Conteo de la frecuencia cardiaca y pulso.

b. Auscultación de frecuencia cardiaca y palpación del pulso simultáneamente.

c. Comparación del pulso: frecuencia, ritmo, igualdad, amplitud y simetría contralateral.

4. Evaluación de la presión arterial:
a. Uso de técnica palpatoria.
b. Uso de técnica auscultatoria.
c. Técnica combinada.
d. Toma de presión arterial en otros sitios.

5. Evaluación de la oximetría de pulso. 


\section{REFERENCIAS}

[1] Ball JW. Manual Seidel de Exploración Física. Barcelona: Elsevier; 2015.

[2] Uribe Mesa AL. Manual para el examen físico del normal y métodos de exploración. Bogotá: CIB; 2010.

[3] Surós Forns J, Surós Batlló A. Surós: semiología médica y técnica exploratoria. Barcelona: Masson; 2001.

[4] Argente Horacio A, Álvarez ME. Semiología médica: fisiopatología, semiotecnia y propedéutica. Enseñanza-aprendizaje centrada en la persona. Buenos Aires: Médica Panamericana; 2013. 


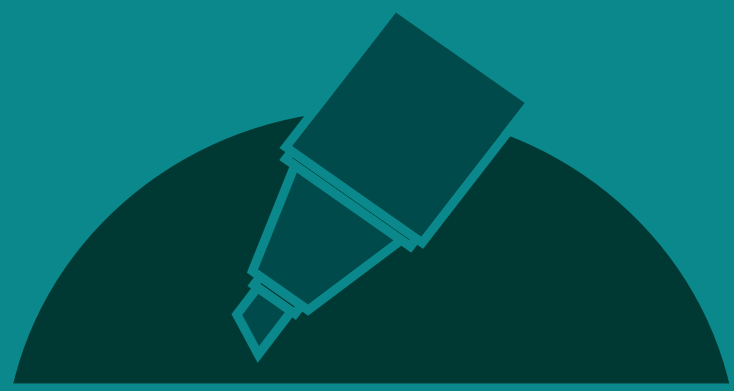

Guías

prácticas 